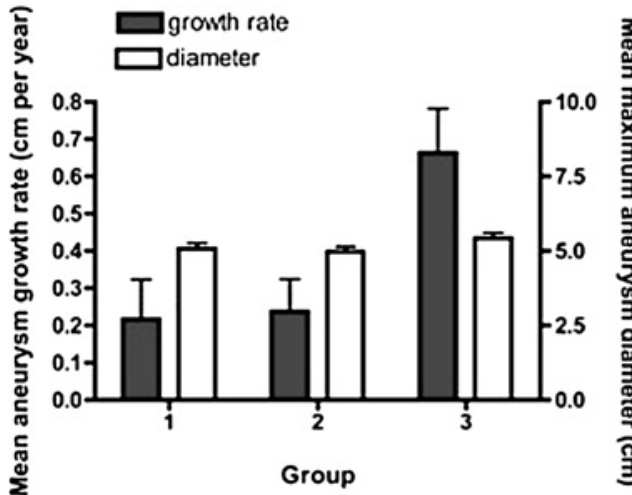

Abstract D Figure 2

\section{INTEGRATIVE GENOMICS APPROACHES IDENTIFY NEW GENES CONTROLLING HEART RATE}

doi:10.1136/heartjnl-2011-300110.5

${ }^{1,2} \mathrm{~J} S$ Ware, ${ }^{3} \mathrm{H}$ Dobrzynski, ${ }^{4} \mathrm{M}$ Pravanec, ${ }^{1} \mathrm{P} \mathrm{J}$ Muckett, ${ }^{1} \mathrm{~S}$ Wilkinson, ${ }^{5} \mathrm{Y}$ Jamshidi, ${ }^{1} \mathrm{~T} J$ Aitma, ${ }^{6} \mathrm{~N}$ S Peters, ${ }^{1,2} \mathrm{~S}$ A Cook. ${ }^{1} \mathrm{MRC}$ Clinical Sciences Centre, Imperial College London, London, UK; ${ }^{2}$ National Heart \& Lung Institute, Imperial College London, London, UK; ${ }^{3}$ School of Medicine, University of Manchester, Manchester, UK; ${ }^{4}$ Institute of Physiology, Czech Academy of Science, Prague, UK; ${ }^{5}$ Division of Clinical Developmental Sciences, St. George's University of London, London, UK; ${ }^{6}$ National Heart \& Lung Institute, Imperial College London, London, UK

Introduction Heart rate (HR) is a fundamental measure of cardiac function, and is of prognostic and therapeutic significance. We applied genetic and genomic approaches to identify new loci and genes controlling HR in a rat model that has previously been used to find human cardiovascular disease genes.

Methods Telemetric aortic pressure transducers were implanted into 226 animals from 33 rat strains: the Brown Norway, the Spontaneously Hypertensive Rat, and 31 strains from a recombinant inbred panel derived from these parental strains and HR was measured over several weeks. Statistical analyses were carried out using the $\mathrm{R}$ package, and quantitative trait loci (OTL) identified by linkage mapping using OTL Reaper. Potential covariates of HR were analysed in SPSS. The sinus node (SN) and right atria (RA) of 20 rats were microdissected (Abstract E Figure 1). Gene expression data were generated with the Affymetrix Rat Gene 1.0 ST microarray and analysed using Bioconductor. Differentially expressed genes were identified using SAM \& Limma. Genes in the QTL that were expressed in the $\mathrm{SN}$ were resequenced to identify potential causative sequence variants.

Results Narrow sense heritability of HR in this population was $51 \%$, suggesting a large genetic contribution to HR. Linkage mapping identified a region on rat chromosome 13 controlling HR, with peak LOD score 6.7 (Abstract E Figure 2A). This QTL has not previously been identified in human, rat or mouse. Mean nocturnal HR in strains carrying the SHR allele was 388, compared with 357 in BN-like strains; an allelic effect of $31 \mathrm{bpm}(8.7 \%, \mathrm{p}<0.00005)$ that is equivalent to $5-9 \mathrm{bpm}$ in humans, corresponding to a decreased risk of cardiovascular death of $10 \%-29 \%$. Two independent approaches (linear regression modelling \& correlation analysis) confirmed that this effect was independent of potential physiological covariates, suggesting that the effect may be intrinsic to the heart, rather than due to neurohumeral influences. We have generated the first genomewide transcript expression profile of the $\mathrm{SN}$. Three genes at the new HR locus were enriched in the SN
(Abstract E Figure 2B) and potentially causative sequence variants in these 3 candidate genes have been identified. We have translated these findings to humans using data from a genome-wide association study population.

Conclusions We have identified a new genomic locus for HR, which does not contain genes in pathways already known to determine HR. We prioritised three candidate genes at the locus, which may be targets for therapeutic modulation of HR in patients with heart disease.

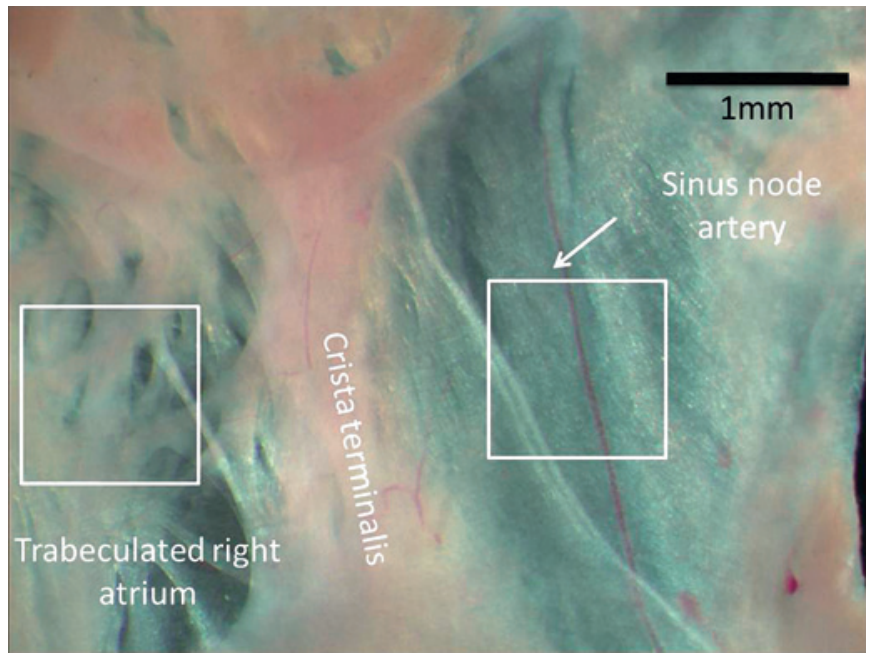

Abstract E Figure 1 Small $\left(1 \mathrm{~mm}^{2}\right)$ pieces of tissue were isolated from the rat SN and distant trabeculated RA and RNA extracted for gene expression profiling.

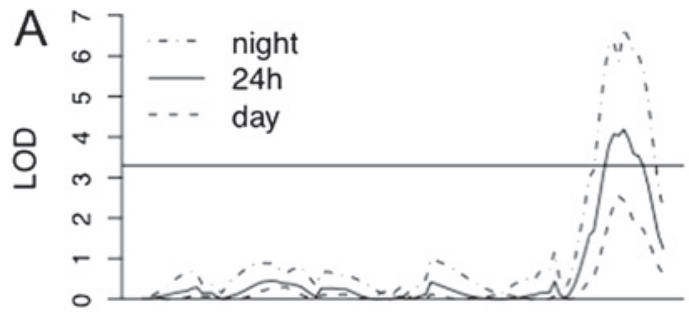

Chromosome 13

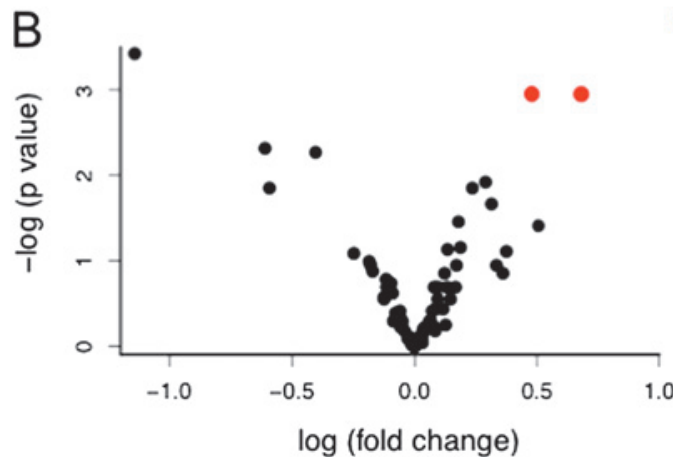

Abstract E Figure 2 (A) Interval mapping revealed a linkage peak on chr13. Linkage was strongest for nocturnal HR, with a LOD score of 6.7 ( $p>0.00005)$. The horizontal line approximates to genomewide significance. (B) A volcano plot showing 3 genes significantly enriched in SN compared with RA. 